\title{
ENIGMA pediatric msTBI: preliminary results from meta-analysis of diffusion MRI
}

Emily L. Dennis, Karen Caeyenberghs, Talin Babikian, Alexander Olsen, Christopher C. Giza, et al.

Emily L. Dennis, Karen Caeyenberghs, Talin Babikian, Alexander Olsen, Christopher C. Giza, Robert F. Asarnow, Harvey Levin, Peter Kochunov, Neda Jahanshad, Paul M. Thompson, David Tate, Elisabeth Wilde, "ENIGMA pediatric msTBI: preliminary results from meta-analysis of diffusion MRI," Proc. SPIE 10975, 14th International Symposium on Medical Information Processing and Analysis, 109750P (21 December 2018); doi: 10.1117/12.2506711

SDIE Event: 14th International Symposium on Medical Information Processing and Analysis, 2018, Mazatlán, Mexico 


\title{
ENIGMA Pediatric msTBI: Preliminary Results from Meta- Analysis of Diffusion MRI
}

Emily L. Dennis ${ }^{1.2}$, Karen Caeyenberghs ${ }^{3}$, Talin Babikian ${ }^{2.4}$, Alexander Olsen ${ }^{2.56}$, Christopher C. Giza' ${ }^{4,7}$, Robert F. Asarnow ${ }^{2,8}$, Harvey Levin', Peter Kochunov ${ }^{10}$, Neda Jahanshad ${ }^{1}$, Paul M. Thompson $^{12,11}$, David Tate ${ }^{12}$, Elisabeth Wilde $e^{1,1,14}$

Imaging Genetics Center, Keck School of Medicine of USC, Marina del Rey, CA, USA; 'Department of Psychiatry and Biobehavioral Sciences, Semel Institute for Neuroscience and Human Behavior, UCLA, Los Angeles, CA, USA; 3 School of Psychology, Faculty of Health Sciences, Australian Catholic University, Melbourne, VIC, Australia; ${ }^{\circ U C L A ~ B r a i n}$ Injury Research Center, Dept of Neurosurgery and Division of Pediatric Neurology, Mattel

Children's Hospital, Los Angeles, CA, USA; 'Department of Psychology, Norwegian

University of Science and Technology, Trondheim, Norway; ${ }^{\circ}$ Department of Physical

Medicine and Rehabilitation, St. Olavs Hospital, Trondheim University Hospital,

Trondheim, Norway; 'Brain Research Institute, UCLA, Los Angeles, CA, USA;

${ }^{\circledR}$ Department of Psychology, UCLA, Los Angeles, CA, USA; 'Department of Physical

Medicine and Rehabilitation, Baylor College of Medicine, Houston, TX, USA; ${ }^{\circ 0}$ University of Maryland School of Medicine, Baltimore, MD; "Departments of Neurology, Pediatrics,

Psychiatry, Radiology, Engineering, and Ophthalmology, USC, Los Angeles, CA;

${ }^{12}$ University of Missouri-St. Louis, St. Louis, MO, USA; ${ }^{13}$ Michael E. DeBakey Veterans

Affairs Medical Center, Houston, TX, USA; ${ }^{4}$ Department of Neurology, University of

Utah, Salt Lake City, UT, USA

\begin{abstract}
Traumatic brain injury (TBI) is a major public health issue around the world. Pediatric TBI patients are at risk of long-term disabilities, as a brain injury sustained during development can affect on-going maturational processes. The white matter (WM) in particular is vulnerable, as myelination continues into the third decade of life and beyond, and poor myelination of tracts can result in decreased integration within brain networks. In addition, variability and heterogeneity are hallmarks of TBI, e.g., injury-related variables and symptoms. These issues combined with small sample sizes limit the power and generalizability of individual studies. In the present study, we employed a meta-analytic approach, combining data across 4 pediatric TBI samples resulting in $104 \mathrm{TBI}(75 \mathrm{M} / 29 \mathrm{~F})$ and 114 control participants $(70 \mathrm{M} / 44 \mathrm{~F})$ between $7-18$ years, using harmonized processing and analysis as part of the ENIGMA consortium (Enhancing NeuroImaging Genetics through MetaAnalysis). We report lower fractional anisotropy (FA) values in TBI patients across several post-injury windows, particularly in central WM tracts. Within the TBI patient group, we also report marginally significant results of lower FA in younger TBI patients, patients scanned closer to time of injury, and female patients. Although this meta-analytic approach yielded the largest sample size reported yet in pediatric moderate/severe TBI (msTBI) neuroimaging, our trends indicate that larger sample sizes are needed in further studies. As additional cohorts join the ENIGMA Pediatric moderate/severe TBI (msTBI) effort, more robust effects will be revealed.
\end{abstract}

Keywords: diffusion magnetic resonance imaging (dMRI), traumatic brain injury (TBI), pediatric, metaanalysis

14th International Symposium on Medical Information Processing and Analysis, edited by Eduardo Romero, Natasha Lepore, Jorge Brieva, Proc. of SPIE Vol. 10975 109750P · (C) 2018 SPIE · CCC code: 0277-786X/18/\$18 - doi: 10.1117/12.2506711 


\section{INTRODUCTION}

Traumatic brain injury (TBI) is the primary cause of death and disability among children and adolescents in the United States (CDC, 2016). More than half a million children experience TBI each year, with the majority being mild injuries. The white matter (WM) is particularly vulnerable to disruption in TBI, and traumatic axonal injury (TAI) is common in more severe injuries. TAI is injury to the axons that occurs when shearing forces during injury cause axons to stretch or break, and via secondary molecular pathways, disrupting communication. Diffusion MRI (dMRI) can offer additional sensitivity to TAI and other WM pathology relative to more conventional imaging methods such as computed tomography (CT) or standard anatomical MRI $[1,2]$. Myelination continues into the third decade of life and beyond, so an injury during development can have serious long-term consequences for brain health [3]. DMRI studies in TBI often show lower fractional anisotropy (FA) post-injury, which is an index of WM organization, although higher FA can be seen immediately post-injury $[1,4]$. Studies investigating the effect of age at injury have found mixed effects $[5,6]$, however these mixed findings could be driven by low sample sizes. There is also a paucity of longitudinal studies of pediatric msTBI using advanced imaging such as dMRI $[2,5,7,8]$.

Even among patients with mild injuries, there is tremendous heterogeneity, with injury severity accounting for a small portion of variation in outcome. Heterogeneity in injury variables, premorbid factors, and recovery environment hamper outcome prediction, and there may be individual variation in biological variables such as inflammatory response [2]. In addition, small sample sizes in brain imaging studies limit the reliability and generalizability. A better understanding of the factors that influence injury and recovery processes will help researchers identify patients at risk for prolonged recovery, and may also indicate potential areas of intervention. The ENIGMA consortium (Enhancing NeuroImaging Genetics through Meta-Analysis) addresses the issue of sample size in imaging studies through harmonized processing and analysis of data across sites and meta-analysis of site-level effects. Here we present preliminary results using the ENIGMA framework to examine 4 cohorts of pediatric moderate/severe TBI (msTBI) across a range of post-injury intervals.

\begin{tabular}{|c|c|c|c|c|}
\hline \multicolumn{5}{|c|}{ Acute } \\
\hline & \multicolumn{2}{|c|}{ BCM1 } & \multicolumn{2}{|c|}{ BCM2 } \\
\hline & TBI & Control & TBI & Control \\
\hline $\mathrm{N}$ & 6 & 3 & 12 & 13 \\
\hline $\mathrm{M} / \mathrm{F}$ & $5 / 1$ & $3 / 0$ & $9 / 3$ & $8 / 5$ \\
\hline Age & $16.6(1.0)$ & $14.1(2.4)$ & $15.4(2.1)$ & $13.1(1.6)$ \\
\hline GCS & $10.0(3.8)$ & NA & $7.7(4.7)$ & NA \\
\hline TSI (weeks) & $3.8(0.6)$ & NA & $3.1(2.0)$ & NA \\
\hline \multicolumn{5}{|c|}{ Post-acute } \\
\hline & \multicolumn{2}{|c|}{ RAPBI } & \multicolumn{2}{|c|}{ BCM3 } \\
\hline & TBI & Control & TBI & Control \\
\hline $\mathrm{N}$ & 27 & 47 & 31 & 39 \\
\hline $\mathrm{M} / \mathrm{F}$ & $19 / 8$ & $23 / 24$ & $22 / 9$ & $27 / 12$ \\
\hline Age & $14.2(2.5)$ & $15.5(2.8)$ & $14.2(2.4)$ & $12.0(2.7)$ \\
\hline GCS & $9(3.8)$ & NA & $6.7(4.1)$ & NA \\
\hline TSI (weeks) & $13.7(4.2)$ & NA & $17.7(3.0)$ & NA \\
\hline \multicolumn{5}{|c|}{ Chronic } \\
\hline & \multicolumn{2}{|c|}{ RAPBI } & \multicolumn{2}{|c|}{ BCM3 } \\
\hline & TBI & Control & TBI & Control \\
\hline $\mathrm{N}$ & 26 & 29 & 34 & 29 \\
\hline $\mathrm{M} / \mathrm{F}$ & $20 / 6$ & $18 / 11$ & $23 / 11$ & $20 / 9$ \\
\hline Age & $16.2(2.3)$ & $16.3(2.8)$ & $14.9(3.0)$ & $13.5(2.8)$ \\
\hline GCS & $9.4(3.7)$ & NA & $7.2(4.3)$ & NA \\
\hline TSI (weeks) & $65.3(7.9)$ & NA & $86.8(13.1)$ & NA \\
\hline
\end{tabular}

Table 1. Demographic information for four cohorts included. Two cohorts contributed data in the acute phase ( $<7$ weeks post-injury, and two contributed data in the post-acute and chronic phases as longitudinal studies. Listed are the size of the TBI and control groups, male/female ratio, age (average and standard deviation), Glasgow Coma Scale for the TBI group (average and SD), and time since injury for the TBI group (in weeks, average and SD).

\subsection{Subjects and Image Acquisition}

Participants were scanned and assessed via

4 different studies, for which the severity ranged between complicated mild and severe TBI. Three sites included children who reported to the emergency room with orthopedic injuries as controls, while the fourth used non-injured children as controls. The post-injury study window varied, therefore participants were clustered into 3 phases: acute ( 0 - 7 weeks post-injury; $18 \mathrm{TBI}$ and 16 controls), post-acute (2-6 months postinjury; $58 \mathrm{TBI}$ and 86 controls), and chronic (12-26 months post-injury; $60 \mathrm{TBI}$ and 58 controls). The RAPBI and BCM3 studies were longitudinal and include data in the post-acute and chronic phases. The studies included are the following: Recovery after Pediatric Brain Injury study from UCLA (RAPBI), the 


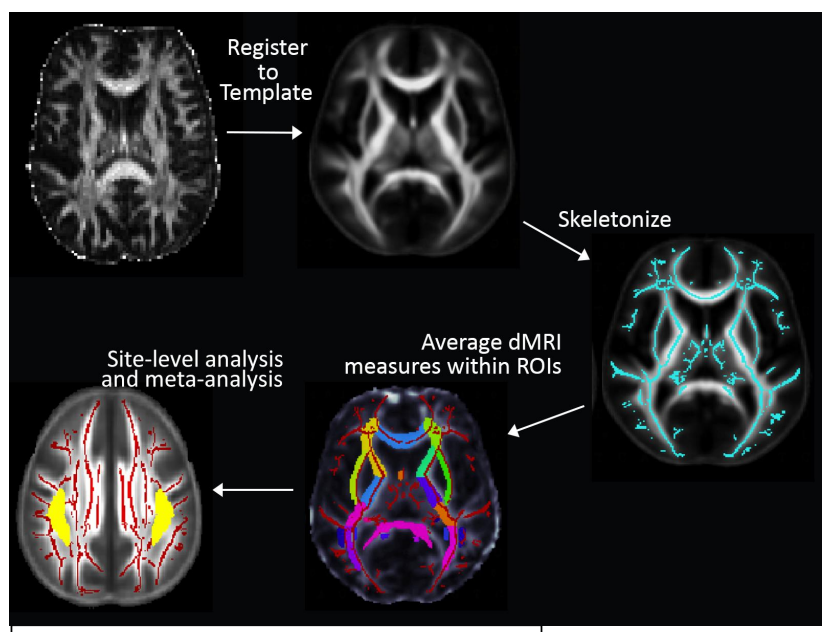

Neurobehavioral Outcome of Head Injury in Children and Evaluation of Mild Head Injury studies of acute pediatric brain injury from Baylor (BCM1 - range 2-7 weeks post-injury, and $\mathrm{BCM} 2$ - range $0-7$ weeks post-injury), and a study of post-acute and chronic imaging markers of msTBI from Baylor (BCM3). Demographic and clinical information for the three cohorts can be seen in Table 1. Raw dMRI data were sent to USC and processed through the standard ENIGMA dMRI protocol, which is based on TBSS (tract-based spatial statistics) in FSL [9] (http://enigma.usc.edu). Scans were eddy corrected, FA, MD (mean diffusivity), RD (radial diffusivity), and $\mathrm{AD}$ (axial diffusivity) images were calculated using FSL dtifit and FA images

Figure 1. ENIGMA dMRI workflow. were used to register data to the ENIGMA-DTI FA template using FSL FLIRT [10]. Each subject's FA values were then projected onto the ENIGMA-DTI FA skeleton; corresponding voxels from diffusion scans were also extracted. Measures were averaged across the entire skeleton (average FA in figures below), and within each of 5 midline, and 19 bilaterally averaged white matter (WM) regions of interest (ROIs) from the Johns Hopkins University (JHU) atlas, some of which partially overlap. Preprocessing included automated and visual quality control of the data at several stages - raw data were examined for artifacts and registration was visually checked. Histograms were generated for each cohort to enable exclusion of outliers. An overview of the method can be seen in Figure 1.

\subsection{Statistics}

TBI/control effect sizes (Cohen's D statistics) were calculated within each site, and statistical results were pooled across sites to conduct a meta-analysis on the individual regression parameters, testing for significant group differences in the $4 \mathrm{dMRI}$ measures averaged within each of the WM ROIs. Our primary model included age and sex as covariates. We conducted additional analyses within the TBI group to examine the effect of age at injury, sex, and TSI (time since injury). Our primary analyses were conducted on FA values, with MD, RD, and $\mathrm{AD}$ serving as post hoc tests. Results were corrected for multiple comparisons using a Bonferroni correction $(p<0.05 / 25=0.002)$.

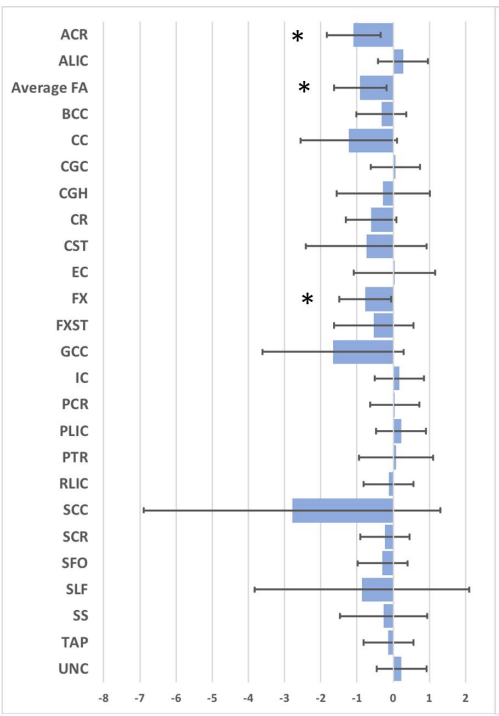

A. Acute

$$
\text { Cohen's D }
$$

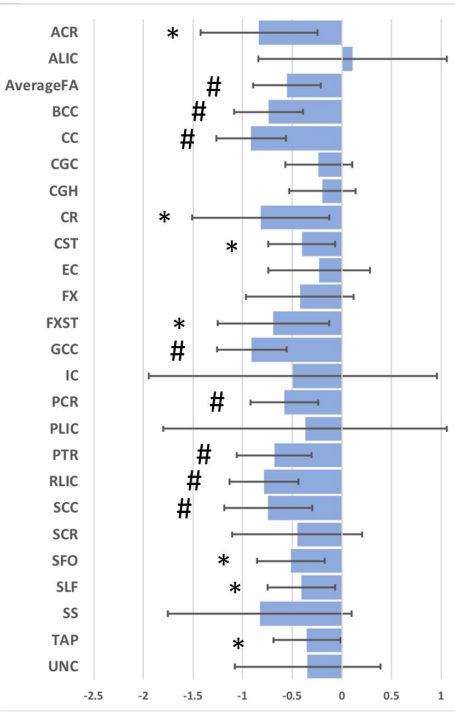

B. Post-acute Cohen's D

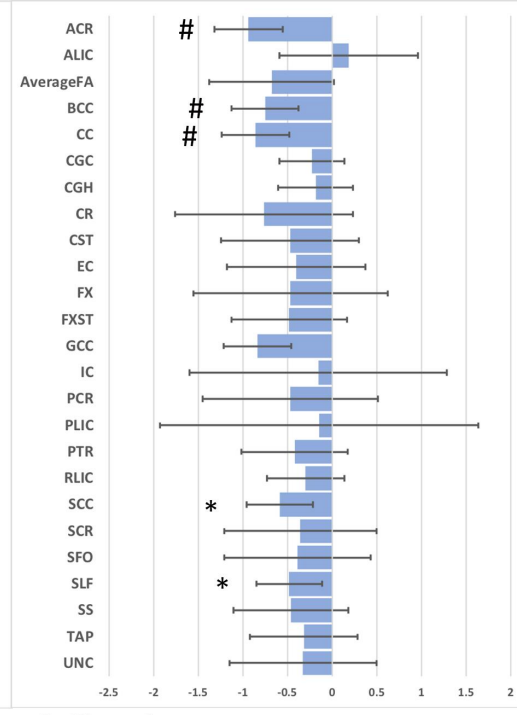

C. Chronic

Cohen's D

Figure 2. Group differences in the A. acute, B. post-acute, and C. chronic phases. Cohen's D statistics are shown across ROIs, with bars indicating 95\% CI. \# denotes significant effects $(p<0.002)$ and $*$ denotes borderline effects $(0.002<p<0.05)$. 


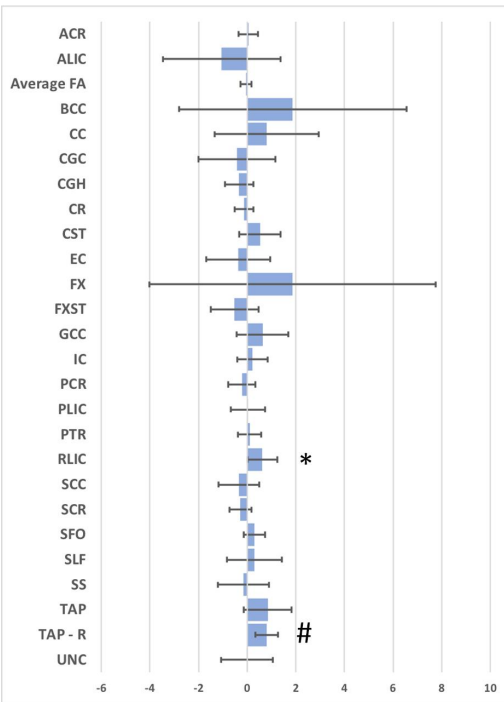

A. Acute

Beta coefficient

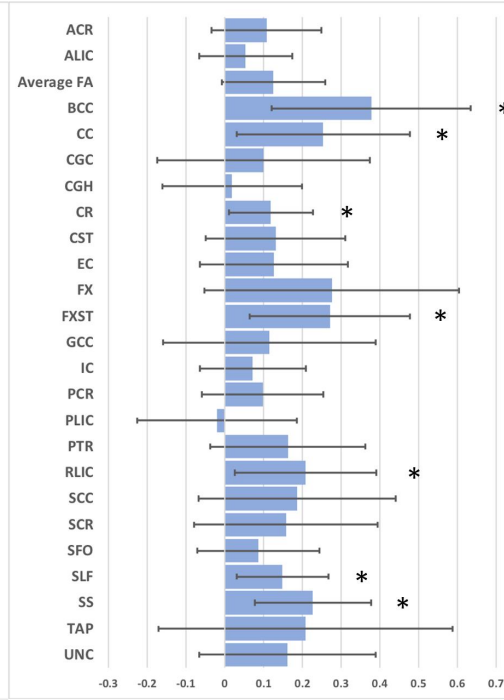

B. Post-acute Beta coefficient

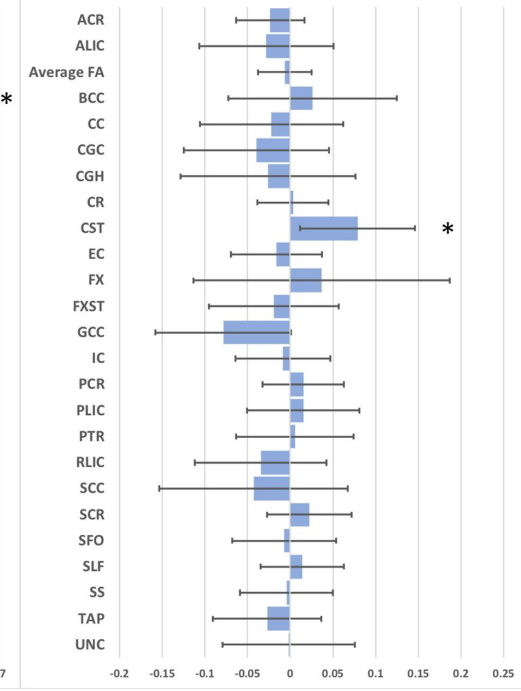

C. Chronic Beta-coefficient

Figure 3. Effect of age-at-injury in the A. acute, B. post-acute, and C. chronic phases. Regression betas from the meta-analysis are shown across ROIs, with bars indicating 95\% CI. \# denotes significant effects $(p<0.002)$ and * denotes borderline effects $(0.002<p<0.05)$.

\section{RESULTS}

\subsection{Acute phase}

In the acute phase, there were no significant differences between the TBI and control groups, with age and sex as covariates. We found marginally significant results $(0.05>p>0.002)$ of lower FA in the TBI group in the corona radiata, fornix, and average FA across the skeleton. These results are depicted in Figure 2A. There was an insufficient sample size to examine sex effects within the TBI group. Examining age-at-injury, including age of scan and sex as covariates, we found a significant effect in the right tapetum and marginally

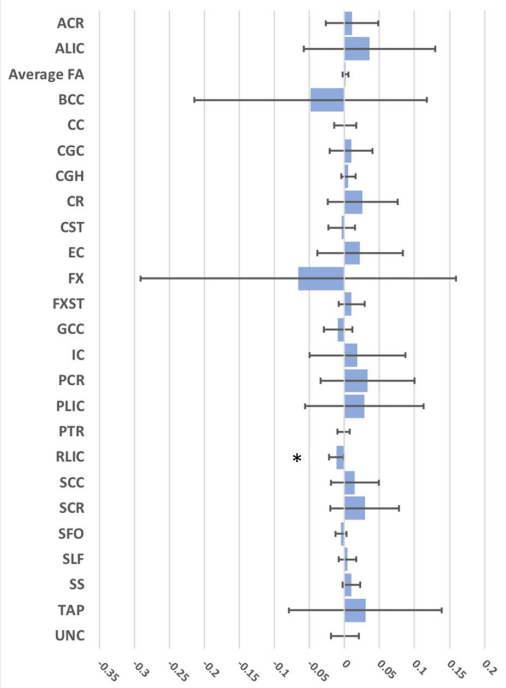

A. Acute Beta coefficient

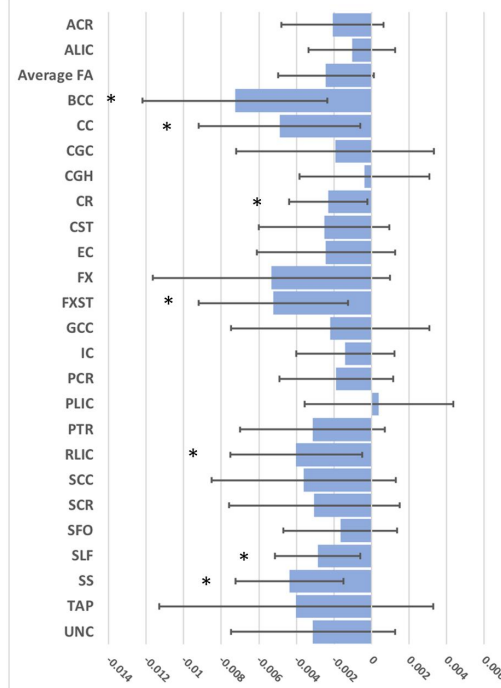

B. Post-acute Beta coefficient

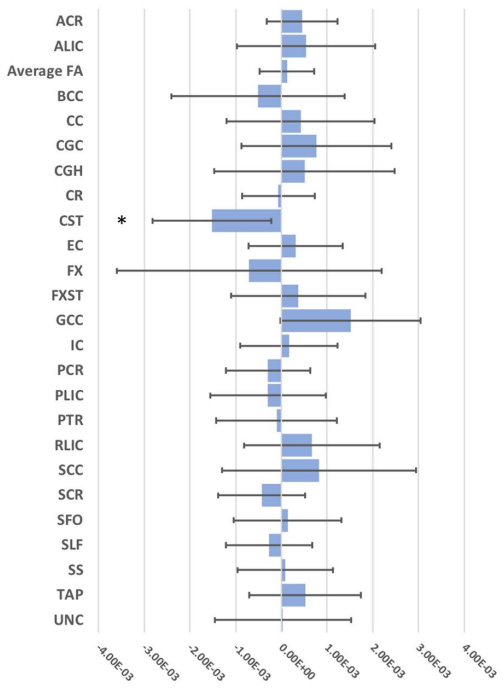

C. Chronic Beta-coefficient

Figure 4. Effect of time since injury (TSI) in the A. acute, B. post-acute, and C. chronic phases. Regression betas from the meta-analysis are shown across ROIs, with bars indicating $95 \% \mathrm{CI} . *$ denotes borderline effects $(0.002<p<0.05)$. 
significant effects in the internal capsule (RLIC, retrolenticular limb), with older patients having higher FA. These results can be seen in Figure 3A. Lastly, within the TBI group we examined the effect of TSI, including age and sex as covariates. As shown in Figure 4A, we found a marginally significant effect in the IC, with patients further from injury having lower FA.

\subsection{Post-acute phase}

In the post-acute phase, we found significantly lower FA in the TBI group across a large number of ROIs, including the corona radiata, corpus callosum, posterior thalamic radiation (PTR), IC, and average FA. Additional ROIs yielded marginally significant lower FA in the TBI group. These results can be seen in Figure 2B. Within the TBI group, we found borderline lower FA in female TBI patients compared to male TBI patients in the uncinate. These effects were not present when comparing control male and female participants. In fact, control males and females showed far more differences, in the same direction, which were not present comparing male and female TBI patients. This could be due in part to larger control samples. These results can be seen in Figure 5A. Examining age-at-injury, including age of scan and sex as covariates, we found marginally significant effects in the superior longitudinal fasciculus, sagittal stratum, corpus callosum, corona radiata, fornix-stria terminalis (FXST), and RLIC. These results can be seen in Figure 3B. Lastly, within the TBI group we examined the effect of TSI, including age and sex as covariates. We found significantly higher FA in patients further from injury in the superior longitudinal fasciculus and sagittal stratum. We additionally found marginally significant effects in the same direction in the corpus callosum, corona radiata, FXST, external capsule, uncinate, IC, PTR, and tapetum. These results can be seen in Figure 4B.

\subsection{Chronic phase}

In the chronic phase, we also found significantly lower FA in the TBI group across several segments of the corona radiata and corpus callosum. Additional ROIs yielded marginally significant lower FA in the TBI group, as seen in Figure 2C. Within the TBI group, we found marginally significant lower FA in female TBI patients compared to male TBI patients in the corpus callosum (body) and corticospinal tract (CST). Within controls, marginally significant sex effects were found in the CST, in addition to several other tracts. These were largely in the same direction apart from the superior fronto-occipital fasciculus, for which control females had higher FA than control males. These results can be seen in Figure 5B. As shown in Figure 3C, we found marginally significant effects in the CST when examining age-at-injury, including age of scan and sex as covariates. Lastly, within the TBI group we examined the effect of TSI, including age and sex as covariates. We found a borderline effect in the CST, with patients further from injury having higher FA. These results can be seen in Figure 4C.
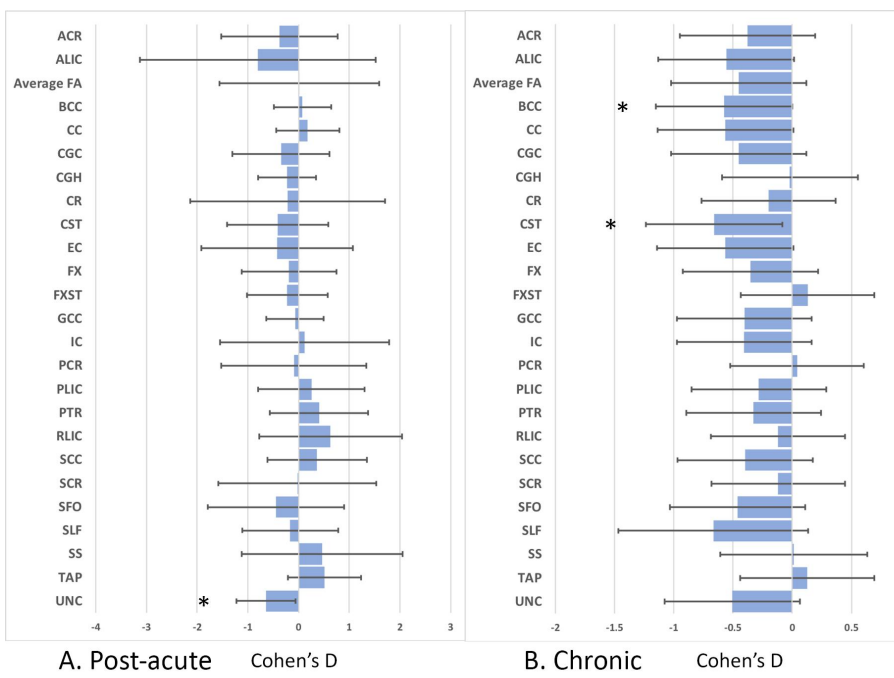

Figure 5. Sex differences within the TBI patient group in the A. postacute and B. chronic phases. Cohen's D are shown across ROIs, with bars indicating $95 \% \mathrm{CI}$ * $*$ denotes borderline effects $(0.002<p<0.05)$.

\section{DISCUSSION}

Here we present preliminary dMRI analyses from the Pediatric msTBI subgroup of the ENIGMA Brain Injury working group. As prior studies have shown, we found lower FA in the TBI patient group, along with some effects of demographic or clinical variables within patients.

Most of the analyses in the acute phase only yielded marginally significant results, with lower FA in the TBI group, and within TBI patients, lower FA in younger patients and those further in the recovery phase. This is likely due to small sample size for this subset. With additional cohorts we may find 
more robust effects. Lower FA post-injury is a consistent finding in pediatric msTBI [4], although higher FA in patients has been reported in the acute phase following a moderate-to-severe injury [1]. Higher FA in patients closer to injury could be due to acute pathology, such as bleeds and edema.

In the post-acute and chronic phases, results were similar, although more extensive and effect sizes larger in the post-acute phase. Fewer results in the chronic phase could indicate some resolution of earlier disruption. We found lower FA in TBI patients compared to controls, especially in central WM tracts such as the corpus callosum and corona radiata. There are several possible explanations for why our effects are localized primarily in central WM. The corpus callosum is particularly vulnerable to injury forces, as the movement of the falx cerebri combined with the weight of both hemispheres places greater strain on the connecting fibers of the corpus callosum [11]. Peripheral tracts may also be affected in a more patient-specific manner, related to the location of lesions. Lastly, reliability of WM measures is best in central WM, particularly when lower resolution dMRI is involved. Future analyses will examine the association between WM disruption and cognitive disruption, with the hypothesis that individual cognitive domains will be associated with specific tract disruptions.

Within the TBI patient group, we found some evidence of an effect of age-at-injury, with younger patients having lower FA. These results were marginally significant, however, and will require larger sample sizes to address in greater detail. We also found marginally significant results suggesting lower FA in patients closer to injury, which could indicate that WM organization recovers over time, although this can only truly be addressed with longitudinal analyses not attempted yet. Lastly, we found some evidence to suggest sex effects, particularly among patients in the chronic phase ( $>12$ months post-injury), with female patients having lower FA than male patients. There have been previous reports of poorer outcome in female concussed athletes compared to male concussed athletes [12], and even some indications of variation within female athletes based on menstrual phase [13], but these studies concern mild TBI, not the more severe injuries included here. There is some animal evidence to suggest that axons in females are more flexible and have fewer microtubules, making them more vulnerable to axonal stretch injury [14]. This question again will require larger sample sizes. An especially interesting question related to the issue of sex differences is the impact of pubertal status on recovery from brain injury.

\section{CONCLUSIONS}

TBI can lead to long-term disruptions to brain health, especially in WM organization. Heterogeneity and small sample sizes have limited the power of prior studies, but by meta-analyzing smaller studies, we can better understand the reliability and generalizability of results. Here we present preliminary results from the ENIGMA Pediatric msTBI working group, using dMRI to examine WM disruption. While our results were largely borderline, with additional cohorts we expect to find more robust effects. Future analyses will also include neurocognitive measures, to identify specific neural disruptions for individual cognitive domains. With larger samples, we hope to identify meaningful patient subtypes that could help develop and evaluate more targeted treatment options.

\section{ACKNOWLEDGMENTS}

ELD is supported by a grant from the NINDS (K99 NS096116). ELD, NJ, PK, and PT are also supported by NIH grants to PT: U54 EB020403, R01 EB008432, R01 AG040060, and R01 NS080655. The RAPBI study (and TB, AO, CCG and RFA) was supported by the NICHD (R01 HD061504). CCG is supported by the UCLA BIRC, NS027544, NS05489, UCLA Steve Tisch BrainSPORT Program and Easton Foundation. The BCM studies (and HL and EW) were supported by NS-21889. 


\section{REFERENCES}

[1] E. L. Dennis, T. Babikian, C. C. Giza et al., "Diffusion MRI in pediatric brain injury," Childs Nerv Syst, 33(10), 1683-1692 (2017).

[2] E. Dennis, T. Babikian, C. Giza et al., "Neuroimaging of the Injured Pediatric Brain: Methods and New Lessons," The Neuroscientist, (2018).

[3] P. Kochunov, D. E. Williamson, J. Lancaster et al., "Fractional anisotropy of water diffusion in cerebral white matter across the lifespan," Neurobiology of Aging, 1-12 (2010).

[4] M. B. Hulkower, D. B. Poliak, S. B. Rosenbaum et al., "A Decade of DTI in Traumatic Brain Injury: 10 Years and 100 Articles Later," AJNR, (2013)

[5] L. Ewing-Cobbs, C. P. Johnson, J. Juranek et al., "Longitudinal diffusion tensor imaging after pediatric traumatic brain injury: Impact of age at injury and time since injury on pathway integrity," Hum Brain Mapp, 37(11), 3929-3945 (2016).

[6] E. L. Dennis, Y. Jin, J. Villalon-Reina et al., "White matter disruption in moderate/severe pediatric traumatic brain injury: advanced tract-based analyses," NeuroImage: Clinical, (2015).

[7] E. L. Dennis; F. Rashid; M. U. Ellis et al, "Diverging White Matter Trajectories in Children after Traumatic Brain Injury: The RAPBI Study," Neurology, 88(15), 1392-1399 (2017).

[8] E. A. Wilde, K. W. Ayoub, E. D. Bigler et al., "Diffusion tensor imaging in moderate-to-severe pediatric traumatic brain injury: changes within an 18 month post-injury interval," Brain Imaging and Behavior, 6(3), 404-416 (2012).

[9] S. M. Smith, M. Jenkinson, H. Johansen-Berg et al., "Tract-based spatial statistics: voxelwise analysis of multi-subject diffusion data," NeuroImage, 31(4), 1487-505 (2006).

[10] J. N. Kelly S, ...(over 60 authors).., Thompson P, Donohoe G, "White matter microstructural differences in 4,072 individuals with schizophrenia versus controls from 28 cohorts worldwide in the ENIGMA Schizophrenia DTI Working Group,” Mol Psychiatry, In Press, (2017).

[11] K. Laksari, M. Kurt, H. Babaee et al., "Mechanistic Insights into Human Brain Impact Dynamics through Modal Analysis," Physical Review Letters, 120(13), 138101 (2018).

[12] D. K. Broshek, T. Kaushik, J. R. Freeman et al., "Sex differences in outcome following sports-related concussion," Journal of Neurosurgery, 102(5), 856-863 (2005).

[13] M. K. Wunderle, K. M. Hoeger, M. E. Wasserman et al., "Menstrual phase as predictor of outcome after mild traumatic brain injury in women," The Journal of Head Trauma Rehabilitation, 29(5), E1 (2014).

[14] J. P. Dolle, A. Jaye, S. A. Anderson et al., "Newfound sex differences in axonal structure underlie differential outcomes from in vitro traumatic axonal injury," Exp Neurol, 300, 121-134 (2018). 